TRANSACTIONS OF THE

AMERICAN MATHEMATICAL SOCIETY

Volume 356, Number 6, Pages 2481-2499

S 0002-9947(04)03379-3

Article electronically published on February 2, 2004

\title{
PRESENTATIONS OF NONEFFECTIVE ORBIFOLDS
}

\author{
ANDRE HENRIQUES AND DAVID S. METZLER
}

\begin{abstract}
It is well known that an effective orbifold $M$ (one for which the local stabilizer groups act effectively) can be presented as a quotient of a smooth manifold $P$ by a locally free action of a compact Lie group $K$. We use the language of groupoids to provide a partial answer to the question of whether a noneffective orbifold can be so presented. We also note some connections to stacks and gerbes.
\end{abstract}

\section{INTRODUCTION}

Recently, motivated largely by string theory, many researchers have developed new aspects of the geometry and topology of orbifolds (e.g. [Rua, [CR, [AR]). In particular, it has become clear that the traditional definition (originally due to Satake Sat56]) is cumbersome and sometimes misleading. Orbifolds should not be seen simply as manifolds with mild singularities (i.e. a very nice kind of stratified space). Instead one wants to take into account the extra structure provided by the stabilizer groups attached to each point.

From the point of view of algebraic geometry this is already known. Orbifolds are the smooth version of Deligne-Mumford stacks. However stacks are not well known outside of algebraic geometry, so this point of view has not been popular among differential geometers or topologists. When one wants to take advantage of the extra structure of an orbifold - for example, to do "stringy geometry" - one must, one way or another, look at the stack structure on the orbifold. Lupercio and Uribe have pointed this out in [LU], although they do not develop it.

More concretely, stacks are represented by groupoids, and it is in this language that many constructions are easier than in the traditional orbifold language. In particular the correct notion of a map of orbifolds corresponds to a Hilsum-Skandalis morphism of groupoids. Such a map induces a continuous map on the underlying topological space, but considering only this continuous map loses information, that is, the functor Orb $\rightarrow$ Top is not faithful. This is the precise sense in which an orbifold has "extra structure." This was only recently observed in the orbifold literature, for example by Ruan and Chen [CR, who noted that to pull back an "orbi-vector bundle" one needs more than a map on the underlying topological space, and by Moerdijk and Pronk [MP97], who observed the same thing for sheaves. See also Hen for concrete examples showing the need for more data.

This paper uses the groupoid language to (partially) answer a question about the presentation of orbifolds. Orbifolds often arise as a quotient of a smooth manifold

Received by the editors February 12, 2003 and, in revised form, April 29, 2003.

2000 Mathematics Subject Classification. Primary 58H05; Secondary 57S10, 18 F99.

Key words and phrases. Orbifolds, groupoids, stacks, gerbes, group actions. 
$P$ by the action of a compact Lie group $K$. Conversely, suppose we require that the stabilizer groups of an orbifold $\mathcal{M}$ act effectively on the corresponding charts. Call such an orbifold effective. (Some sources refer to these as reduced orbifolds, but this conflicts with the standard use of the word "reduced" in algebraic geometry.) Then any effective $\mathcal{M}$ can be presented 1 as $\mathcal{M} \cong P / K$ for some $P, K$.

If one thinks of an orbifold as simply a mildly singular stratified space, with the main function of the stabilizer groups being to provide interesting singularities, then the effectivity assumption is reasonable. However when one wants to utilize the extra structure associated to the stabilizer groups, this restriction becomes artificial. In addition, certain useful constructions on orbifolds (such as Kawasaki's $\tilde{M}$, Kaw78, [Kaw79]) take one out of the effective situation.

Even the extreme case where the actions of the stabilizer groups are all trivial turns out to be interesting; the presentation problem here is the topological analogue of the Brauer Conjecture (see [EHKV]) in algebraic geometry. We will see that the appropriate language for this case uses gerbes.

Hence a natural question arises, can a noneffective orbifold always be presented? The answer is still unknown, but we prove results (Theorem 5.5, Corollary [5.6 and Theorem 5.7 below) showing that in many cases the answer is still yes. The extension of our method to answer the general question will require understanding equivariant gerbes.

1.1. Usual definition of an orbifold. For completeness, we recall the usual definition of an orbifold. We adapt the following from Ruan and Chen [CR]. (See also Moerdijk and Pronk [MP97.) We note that in Section 2.4 we will see an alternate characterization of orbifolds, one that we think of as a superior definition. Let $M$ be a paracompact Hausdorff space.

Definition 1.1. An orbifold chart on $M$ is given by a connected open subset $\tilde{U} \subset \mathbb{R}^{n}$, an action $\rho: H \rightarrow \operatorname{Aut}(\tilde{U})$ of a finite group $H$ by smooth automorphisms of $\tilde{U}$, and a map $\phi: \tilde{U} \rightarrow M$, such that $\phi$ is $H$-invariant and induces a homeomorphism from $\tilde{U} / H$ onto an open subset $U=\phi(\tilde{U}) \subset M$.

An embedding $\lambda:(\tilde{U}, H, \rho, \phi) \rightarrow\left(\tilde{U}^{\prime}, H^{\prime}, \rho^{\prime}, \phi^{\prime}\right)$ between two such charts is a smooth embedding $\lambda: \tilde{U} \rightarrow \tilde{U}^{\prime}$ and an injective map, also denoted by $\lambda, \lambda: H \rightarrow$ $H^{\prime}$, with $\lambda(h \cdot \tilde{x})=\lambda(h) \cdot \lambda(\tilde{x})$, and $\phi(\lambda(\tilde{x}))=\phi^{\prime}(\tilde{x})$. Furthermore, $\lambda: H \rightarrow H^{\prime}$ is required to be an isomorphism from the kernel of the action map $H \rightarrow \operatorname{Aut}(\tilde{U})$ to the kernel of $H^{\prime} \rightarrow \operatorname{Aut}\left(\tilde{U}^{\prime}\right)$.

An orbifold atlas on $M$ is a family $\mathcal{U}=\left\{\left(\tilde{U}_{\alpha}, H_{\alpha}, \rho_{\alpha}, \phi_{\alpha}\right)\right\}$ of such charts, which cover $M$ and are compatible in the following sense: given any two charts $\left(\tilde{U}_{\alpha}, H_{\alpha}, \rho_{\alpha}, \phi_{\alpha}\right)$ and $\left(\tilde{U}_{\beta}, H_{\beta}, \rho_{\beta}, \phi_{\beta}\right)$, with $\phi_{\alpha}\left(\tilde{U}_{\alpha}\right)=U_{\alpha}, \phi_{\beta}\left(\tilde{U}_{\beta}\right)=U_{\beta}$, and given any $x \in U_{\alpha} \cap U_{\beta}$, there exists an open neighborhood $U_{\gamma} \subset U_{\alpha} \cap U_{\beta}$ and a chart $\left(\tilde{U}_{\gamma}, H_{\gamma}, \rho_{\gamma}, \phi_{\gamma}\right)$ for $U_{\gamma}$, such that there are embeddings $\left(\tilde{U}_{\gamma}, H_{\gamma}, \rho_{\gamma}, \phi_{\gamma}\right) \rightarrow$ $\left(\tilde{U}_{\alpha}, H_{\alpha}, \rho_{\alpha}, \phi_{\alpha}\right)$ and $\left(\tilde{U}_{\gamma}, H_{\gamma}, \rho_{\gamma}, \phi_{\gamma}\right) \rightarrow\left(\tilde{U}_{\beta}, H_{\beta}, \rho_{\beta}, \phi_{\beta}\right)$.

Two such atlases are said to be equivalent if they have a common refinement. An orbifold (of dimension $n$ ) is such a space $M$ with an equivalence class of orbifold atlases.

\footnotetext{
${ }^{1}$ This is very different from asking whether an orbifold can be obtained as a quotient by the action of a finite group, or more generally a discrete group. So this question has nothing to do with the distinction between "good" and "bad" orbifolds.
} 
Many authors consider only effective (or "reduced") orbifolds, defined as follows.

Definition 1.2. An orbifold is effective if it has an atlas in which all of the group actions $\rho_{\alpha}$ are effective.

That is, for effective orbifolds, we can think of each stabilizer group as a subgroup of $\operatorname{Aut}(\tilde{U})$.

For a noneffective orbifold, the role of the noneffective parts of the stabilizer groups (the kernels of the maps $\rho_{\alpha}$ ) is rather subtle. In Section 2.4 we will use the language of groupoids to give an alternate characterization of an orbifold, which agrees with the above definition in the effective case. It is the groupoid definition which we will work with in the sequel.

One obvious way to get an orbifold is to start with a smooth manifold and mod out by the action of a finite group. It is simple to find examples of orbifolds which do not arise in this way. A more general situation in which orbifolds arise is the following easy consequence of the slice theorem for group actions:

Proposition 1.3. Let $P$ be a smooth manifold with a locally free action of a compact Lie group $K$. (I.e. the stabilizers are all finite.) Then the quotient $P / K$ has a canonical orbifold structure.

When an orbifold $M$ can be realized as such a quotient $M \cong P / K$, we say that this is a presentation of $M$. In the paper [Sat56] which introduced orbifolds (under the name "V-manifolds") Satake proved the following result about presentations.

Proposition 1.4. Let $M$ be an effective orbifold. Then $M$ can be presented as $M \cong$ $P / K$, where $P=\operatorname{Fr}_{O(n)}(M)$ is the orthonormal frame bundle for some Riemannian structure on $M$ and $K=O(n)$.

The purpose of this paper is to generalize this result to a larger class of orbifolds than the effective ones, and give partial results toward the presentation question for all orbifolds.

\section{Groupoids}

As mentioned in the Introduction, one of the ways to think of an orbifold is as a particularly simple kind of smooth groupoid. We will briefly review the notion of groupoid, and introduce the structures on groupoids which we will need in the sequel. A good source for some of the material in this section is the recent preprint of Moerdijk [Moe. In Section 2.4 we will address how to think of orbifolds as groupoids.

A groupoid is a category where all morphisms are invertible. We need the notion of a smooth groupoid, where the sets of objects and of arrows are smooth manifolds, and the structure maps of the groupoid are smooth maps 2 More precisely,

Definition 2.1. A smooth groupoid $\mathcal{G}$ is a pair of smooth manifolds $\mathcal{G}_{1}, \mathcal{G}_{0}$ and smooth maps $s, t: \mathcal{G}_{1} \rightarrow \mathcal{G}_{0}, u: \mathcal{G}_{0} \rightarrow \mathcal{G}_{1}, i: \mathcal{G}_{1} \rightarrow \mathcal{G}_{1}, m: \mathcal{G}_{1} \times{ }_{s, \mathcal{G}_{0}, t} \mathcal{G}_{1} \rightarrow \mathcal{G}_{1}$, satisfying the relations given below. We call $\mathcal{G}_{0}$ the space of objects and $\mathcal{G}_{1}$ the space of arrows. We use the notation $g: x \rightarrow y$ to denote an arrow $g \in \mathcal{G}_{1}$ with

\footnotetext{
${ }^{2}$ One can also work in the topological category. Here we restrict ourselves to the smooth setting.
} 
$s(g)=x, t(g)=y$. Denoting $m(g, h)$ by $g h, i(g)$ by $g^{-1}$, and $u(x)$ by $1_{x}$, the axioms are the familiar ones for a groupoid: for appropriate $x, y \in \mathcal{G}_{0}, g, h, k \in \mathcal{G}_{1}$,

$$
\begin{gathered}
s\left(1_{x}\right)=t\left(1_{x}\right)=x, \quad s\left(g^{-1}\right)=t(g), \quad t\left(g^{-1}\right)=s(g) \\
s(g h)=s(h), \quad t(g h)=t(g), \quad g(h k)=(g h) k \\
g 1_{x}=1_{y} g=g, \quad g^{-1} g=1_{x}, \quad g g^{-1}=1_{y} \quad(\text { for } g: x \rightarrow y) .
\end{gathered}
$$

We further require that $s$ (and hence $t$ ) is a submersion (in particular, this guarantees that $\mathcal{G}_{1} \times{ }_{s, \mathcal{G}_{0}, t} \mathcal{G}_{1}$ is actually a smooth manifold). Note: we do require all manifolds to be Hausdorff (and second countable).

If $s$ (hence $t$ ) is a local diffeomorphism, we call $\mathcal{G}$ a smooth étale groupoid. If the map $(s, t): \mathcal{G}_{1} \rightarrow \mathcal{G}_{0} \times \mathcal{G}_{0}$ is proper, we call the groupoid proper.

Note: It is easy to show that $i$ is an involution of $\mathcal{G}_{1}$; hence any property holds for $s$ if and only if it holds for $t$.

When we want to refer to a groupoid without any topological or smooth structure, we will use the term abstract groupoid.

Examples. (1) Given a manifold $M$, we can define the trivial groupoid, also denoted $M$, with $M_{0}=M$ and with only identity arrows in $M_{1}$.

(2) Given a Lie group $K$ we can define a corresponding groupoid $\mathcal{B} K$ with only one object, and with $K$ as the space of arrows.

(3) Given a manifold $M$ and a right action of the Lie group $K$ on $M$, we can form the translation groupoid $M \rtimes K$ by $(M \rtimes K)_{0}=M,(M \rtimes K)_{1}=M \times K$, $s(x, k)=x k, t(x, k)=x, u(x)=(x, 1), i(x, k)=\left(x k, k^{-1}\right)$, and $(x, k) \circ(y, h)=$ $(x, k h)$.

Note that the first example is $M \rtimes(e)$, and the second example is $* \rtimes K$, where $*$, here and in the sequel, denotes the one-point space. The notation $\mathcal{B K}$ is meant to be suggestive of classifying spaces; see Section 2.3 for a justification of this notation.

A strict homomorphism $\phi: \mathcal{G} \rightarrow \mathcal{H}$ of smooth groupoids is a smooth functor, i.e. it is given by a pair of smooth maps $\phi_{0}: \mathcal{G}_{0} \rightarrow \mathcal{H}_{0}$ and $\phi_{1}: \mathcal{G}_{1} \rightarrow \mathcal{H}_{1}$ commuting with the structure maps of $\mathcal{G}$ and $\mathcal{H}$. We get a corresponding notion of strict isomorphism. However we will use a different notion of morphism, explained in Section 2.3 below.

Given any smooth groupoid $\mathcal{G}$, one can form its topological, or coarse, quotient $\mathcal{G}_{\text {top }}=\mathcal{G}_{0} / \mathcal{G}$ by identifying any two objects which have an arrow between them. However this is not a very fine invariant of $\mathcal{G}$ and is often not a nice topological space. For example, any groupoid that is categorically connected (i.e., any two objects can be joined by an arrow) has a topological quotient that is just a single point. Note that if we take the topological quotient of a translation groupoid $X \rtimes K$, we get the topological quotient: $(X \rtimes K)_{\text {top }} \cong X / K$. We will think of groupoids as models for their quotient spaces which are finer than the naïve topological quotient. The precise way in which we use a groupoid as a model for its quotient comes from the notion of map which we will define in Section 2.3.

2.1. Structures on groupoids. Most notions that can be defined for a group or for a manifold can be defined for groupoids. We will need the following notions.

Let $\mathcal{G}$ be a groupoid. A right action of $\mathcal{G}$ on a space $X$ consists of two smooth maps $\pi: X \rightarrow \mathcal{G}_{0}$ (often called the "moment map"; we will call it the "base map" 
to avoid confusion with symplectic geometry usage) and

$$
\begin{aligned}
m: X \times_{\pi, \mathcal{G}_{0}, t} \mathcal{G}_{1}=\{(x, g) \mid \pi(x)=t(g)\} & \rightarrow X, \\
(x, g) & \mapsto x g
\end{aligned}
$$

(the action map) such that

$$
(x g) h=x(g h), \quad x 1=x, \quad \pi(x g)=s(g) .
$$

Given a right action of $\mathcal{G}$ on $X$ we can form the semidirect product, or translation, groupoid, generalizing the third example from the previous section: we define

$$
(X \rtimes \mathcal{G})_{0}=X, \quad(X \rtimes \mathcal{G})_{1}=X \times_{\pi, \mathcal{G}_{0}, t} \mathcal{G}_{1}
$$

and $s(x, g)=x g, t(x, g)=x,(x, g)(x g, h)=(x, g h), i(x, g)=\left(x g, g^{-1}\right)$. Note that $\pi$ extends as a covariant functor $\pi: X \rtimes \mathcal{G} \rightarrow \mathcal{G}$ by defining $\pi(x, g)=g$. Unless otherwise specified all actions will be from the right, so by a $\mathcal{G}$-space we will mean a space with a given right $\mathcal{G}$-action.

A left action of $\mathcal{G}$ is a right action of the opposite groupoid $\mathcal{G}^{\text {op }}$. We will use this a good deal so we will be explicit. We have a base map $\pi: X \rightarrow \mathcal{G}_{0}$ and an action map

$$
\begin{aligned}
m: \mathcal{G}_{1} \times_{s, \mathcal{G}_{0}, \pi} X=\{(g, x) \mid s(g)=\pi(x)\} & \rightarrow X, \\
(g, x) & \mapsto g x
\end{aligned}
$$

(the action map) such that

$$
g(h x)=(g h) x, \quad 1 x=x, \quad \pi(g x)=t(g) .
$$

Again we can form a semidirect product:

$$
(\mathcal{G} \ltimes X)_{0}=X, \quad(\mathcal{G} \ltimes X)_{1}=\mathcal{G}_{1} \times_{s, \mathcal{G}_{0}, \pi} X
$$

and $s(g, x)=x, t(g, x)=g x,(g, h x)(h, x)=(g h, x), i(g, x)=\left(g^{-1}, g x\right)$. With these definitions $\pi$ again extends as a covariant functor $\pi: \mathcal{G} \ltimes X \rightarrow \mathcal{G}$ by defining $\pi(g, x)=g$.

A $\mathcal{G}$-sheaf of sets (or $\mathcal{G}$-equivariant sheaf, or just sheaf) over $\mathcal{G}$ is a $\mathcal{G}$-space $E$ such that the base map $\pi: E \rightarrow \mathcal{G}_{0}$ is étale (i.e. a local diffeomorphism, not necessarily surjective). Sheaves of groups, covering spaces, and local systems (i.e. locally constant sheaves) are defined similarly.

We will need the following definition to describe maps of groupoids in the next section.

Definition 2.2. Given a manifold $B$, a (right) $\mathcal{G}$-bundle over $B$ is a (right) $\mathcal{G}$-space $E$ and a smooth $\mathcal{G}$-invariant map $p: E \rightarrow B($ so $p(x g)=p(x))$. It is principal if $p$ is a surjective submersion and

$$
E \times \times_{\mathcal{G}_{0}} \mathcal{G}_{1} \rightarrow E \times \times_{B} E, \quad(e, g) \mapsto(e, e g)
$$

is a diffeomorphism.

This reduces to the usual notion of a principal bundle in the case of a Lie group, i.e. when $\mathcal{G}_{0}$ is a point.

In Section [5, to make certain reductions in the presentation problem, we will need the notion of a group action on a groupoid and the resulting semidirect product. (One can in fact define the notion of a groupoid acting on a groupoid, but we will not need this notion.) 
Let $\mathcal{G}$ be a smooth groupoid and let $K$ be a Lie group. A right action of $K$ on $\mathcal{G}$ is a right action (in the usual sense) of $K$ on $\mathcal{G}_{0}$ and a right action of $K$ on $\mathcal{G}_{1}$ which respect the structure maps of $\mathcal{G}$, namely,

$u(x) \cdot k=u(x \cdot k), \quad s(g \cdot k)=s(g) \cdot k, \quad t(g \cdot k)=t(g) \cdot k, \quad(g h) \cdot k=(g \cdot k)(h \cdot k)$.

Note that every element of $K$ acts by an automorphism of the groupoid $\mathcal{G}$.

(Note: this is a very strict notion of a group action on a groupoid; one could define looser "2-categorical" notions but we will not need them.)

Given $K$ acting on $\mathcal{G}$ on the right, we define the semidirect product groupoid by

$$
(\mathcal{G} \rtimes K)_{0}=\mathcal{G}_{0}, \quad(\mathcal{G} \rtimes K)_{1}=\mathcal{G}_{1} \times K
$$

with $s(g, k)=s(g) \cdot k, t(g, k)=t(g)$, and $(g, k)\left(g^{\prime}, k^{\prime}\right)=\left(g\left(g^{\prime} \cdot k^{-1}\right), k k^{\prime}\right)$. This reduces to the usual semidirect product of groups when $\mathcal{G}$ is a group, i.e. when it has just one object; and it reduces to the previous definition of semidirect product when $\mathcal{G}$ is a space, i.e. when it has only identity arrows.

We have the following useful lemma about semidirect products:

Lemma 2.3. Let $\mathcal{G}$ be a smooth groupoid and let $K, L$ be two Lie groups acting on $\mathcal{G}$ on the right. Assume that the actions commute. Then there is a natural strict isomorphism

$$
(\mathcal{G} \rtimes K) \rtimes L \cong \mathcal{G} \rtimes(K \times L) .
$$

2.2. Stabilizers. We will be particularly interested in the following $\mathcal{G}$-spaces. The stabilizer space $S_{\mathcal{G}}$ of a groupoid $\mathcal{G}$ (denoted simply by $S$ when it does not cause confusion) is defined to be the set of arrows with the same source and target:

$$
S_{\mathcal{G}}=\left\{g \in \mathcal{G}_{1} \mid s(g)=t(g)\right\} .
$$

(In categorical language, these are the automorphisms.) There is a right action of $\mathcal{G}$ by conjugation: given $g \in \mathcal{G}_{1}, h \in S_{\mathcal{G}}$, we then define $h \cdot g=g^{-1} h g$. Hence $S_{\mathcal{G}}$ is a $\mathcal{G}$-space. Given $x \in \mathcal{G}_{0}$, let $S_{x}=\{g: x \rightarrow x\}$ be the space of stabilizer arrows at $x$; if $\mathcal{G}$ is étale, then $S_{x}$ is discrete.

Let $\mathcal{G}$ be a smooth étale groupoid. An arrow $g: x \rightarrow y$ induces a germ of a diffeomorphism $\phi_{g}$ from $x$ to $y$. Choose an open neighborhood $U$ of $g$ such that $\left.s\right|_{U}: U \rightarrow s(U) \subset \mathcal{G}_{0}$ is a diffeomorphism. Then we have a smooth map $t \circ\left(\left.s\right|_{U}\right)^{-1}: s(U) \rightarrow t(U)$, such that $x \mapsto y$. Different choices of $U$ give the same germ. This defines a strict homomorphism $\phi: \mathcal{G} \rightarrow \operatorname{Diff}\left(\mathcal{G}_{0}\right)$, the latter being the groupoid of germs of diffeomorphisms of $\mathcal{G}_{0}$.

In particular, given a stabilizer arrow $(g: x \rightarrow x) \in S$, consider the germ $\phi_{g}$, which is the germ of a diffeomorphism fixing $x$. The space of ineffective stabilizers $S^{0}=S_{\mathcal{G}}^{0}$ is defined as the subspace of $S$ consisting of the arrows which induce the trivial germ (the germ of the identity map) on $\mathcal{G}_{0}$. Let $S_{x}^{0}=S_{\mathcal{G}}^{0} \cap S_{x}$ be the set of ineffective stabilizers at $x$.

Proposition 2.4. Let $\mathcal{G}$ be an étale groupoid. Then $S_{\mathcal{G}}^{0}$ is an open and closed $\mathcal{G}$-equivariant subspace of $\mathcal{G}_{1}$. Hence $S_{\mathcal{G}}^{0}$ is a $\mathcal{G}$-sheaf. If $\mathcal{G}$ is proper, then $S_{\mathcal{G}}^{0}$ is a local system.

Proof. First note that an arrow $g \in S_{x}$ is in $S_{\mathcal{G}}^{0}$ if and only if there is a neighborhood $U \subset \mathcal{G}_{1}$ of $g$ such that $\left.s\right|_{U}=\left.t\right|_{U}$. Given such a $U$, every $h \in U$ is also in $S_{\mathcal{G}}^{0}$, so $S_{\mathcal{G}}^{0}$ is open. Also, $S_{\mathcal{G}}^{0}$ is the inverse image under $\phi$ of the identity arrows in $\operatorname{Diff}\left(\mathcal{G}_{0}\right)$, so it is closed as well. 
Now we show that $S_{\mathcal{G}}^{0}$ is $\mathcal{G}$-equivariant. Given an arrow $g \in S_{x}^{0}$, let $U$ be such that $\left.s\right|_{U}=\left.t\right|_{U}$ and there is a section $a: V=s(U) \rightarrow U$ of $s=t$. Let $h: y \rightarrow x$ and, making $V$ smaller if necessary, let $b: V \rightarrow \mathcal{G}_{1}$ be a section of $t$ such that $b(x)=h$. Let $c: V \rightarrow \mathcal{G}_{1}$ be defined by $c(z)=b(z)^{-1} a(z) b(z)$. It is easy to see that $s(c(z))=t(c(z))$, that $c(x)=h^{-1} g h$, and that $c(V)$ is open in $\mathcal{G}_{1}$. Hence the conjugate $h^{-1} g h$ is also in $S_{\mathcal{G}}^{0}$.

Now suppose that $\mathcal{G}$ is proper. We claim that $s=t: S_{\mathcal{G}}^{0} \rightarrow \mathcal{G}_{0}$ is proper. Let $K \subset \mathcal{G}_{0}$ be compact. Then

$$
s^{-1}(K) \cap S_{\mathcal{G}}^{0}=(s, t)^{-1}(K \times K) \cap S_{\mathcal{G}}^{0}
$$

is the intersection of a compact and a closed set, hence is compact. Now we claim that $s: S_{\mathcal{G}}^{0} \rightarrow \mathcal{G}_{0}$ is a covering map, which will complete the proof. This follows from the following general topology lemma.

Lemma 2.5. Let $p: E \rightarrow X$ be a proper étale map. Assume that $X$ is locally compact and $E$ is Hausdorff. Then $p$ is a covering map.

Proof. Let $x \in X$. Since $p$ is proper, $p^{-1}(x)=\left\{e_{1}, \ldots, e_{n}\right\}$ is finite, and each $e_{k}$ has a neighborhood $U_{k}$ such that $\left.p\right|_{U_{k}}$ is a diffeomorphism onto its image. Since $E$ is Hausdorff, we can assume that the $U_{k}$ are disjoint. Let $U=\coprod U_{k}$ and let $V=\bigcap p\left(U_{k}\right)$. Since $X$ is locally compact, there is a neighborhood $W$ of $x$ with $\bar{W} \subset V$ compact. Let

$$
A=p^{-1}(\bar{W}) \backslash U \subset E,
$$

which is compact, and let $B=p(A) \subset X$, also compact, hence closed. Since $A \cap p^{-1}(x)=\emptyset, x \notin B$, so there is a neighborhood $W^{\prime}$ of $x$ with $W^{\prime} \subset \bar{W}$ and $W^{\prime} \cap B=\emptyset$. Then $p^{-1}\left(W^{\prime}\right) \subset U$, so $p^{-1}\left(W^{\prime}\right)=\coprod U_{k}^{\prime}$ for some open subsets $U_{k}^{\prime} \subset U_{k}$. Hence $W^{\prime}$ is evenly covered, and $p$ is a covering map.

If $S_{\mathcal{G}}^{0}$ consists only of identity arrows, we say $\mathcal{G}$ is effective. In this paper we will be most concerned with noneffective groupoids.

Given any groupoid $\mathcal{G}$ we can quotient out by the ineffective stabilizers. We define

$$
\left(\mathcal{G}_{\text {eff }}\right)_{0}=\mathcal{G}_{0} \quad \text { and } \quad\left(\mathcal{G}_{\text {eff }}\right)_{1}=\mathcal{G}_{1} / S_{\mathcal{G}}^{0}
$$

Since $S_{\mathcal{G}}^{0}$ is $\mathcal{G}$-equivariant and acts trivially on $\mathcal{G}_{0}, \mathcal{G}_{\text {eff }}$ is a smooth effective groupoid. If $\mathcal{G}$ is étale (resp. proper), then so is $\mathcal{G}_{\text {eff }}$. There is an evident strict homomorphism $p: \mathcal{G} \rightarrow \mathcal{G}_{\text {eff }}$; two elements $g, h: x \rightarrow y \in \mathcal{G}_{1}$ have $p(g)=p(h)$ if they induce the same germ of a diffeomorphism from $x$ to $y$.

2.3. Maps of groupoids. We have already defined the notion of a strict homomorphism of groupoids. However, we will more often use a different notion of morphism of groupoids. Since we are only interested in $\mathcal{G}$ as a model for its quotient, we need to be looser about what a morphism can be. The appropriate notion of map is that of Hilsum and Skandalis. We follow Crainic [Cra99] here. The key is that we are (roughly) allowed to "replace" the source groupoid by an "equivalent" object. Here the equivalent object is a principal bundle over the space of objects of the source.

Definition 2.6. Let $\mathcal{G}$ and $\mathcal{H}$ be smooth groupoids. A (Hilsum-Skandalis) morphism from $\mathcal{G}$ to $\mathcal{H}$ consists of a manifold $P$, maps $s_{P}: P \rightarrow \mathcal{G}_{0}, t_{P}: P \rightarrow \mathcal{H}_{0}$, a left action of $\mathcal{G}$ on $P$ with the base map $s_{P}$, a right action of $\mathcal{H}$ on $P$ with base map $t_{P}$, such that:

(1) $s_{P}$ is $\mathcal{H}$-invariant, $t_{P}$ is $\mathcal{G}$-invariant; 
(2) the actions of $\mathcal{G}$ and $\mathcal{H}$ on $P$ are compatible: given $p \in P,(g p) h=g(p h)$;

(3) $s_{P}: P \rightarrow \mathcal{G}_{0}$, as an $\mathcal{H}$-bundle with base map $t_{P}$, is principal.

We will often abuse notation and denote such a morphism simply by $P$.

When we use the word "morphism" or "map" without qualification, we will always mean "Hilsum-Skandalis morphism."

The composition of two morphisms $P: \mathcal{G} \rightarrow \mathcal{H}$ and $Q: \mathcal{H} \rightarrow \mathcal{K}$ is defined by dividing out $P \times \mathcal{H}_{0} Q$ by the action of $\mathcal{H}:(p, q) h=\left(p h, h^{-1} q\right)$, and taking the obvious actions of $\mathcal{G}$ and $\mathcal{K}$. The identity map of $\mathcal{G}$ is represented by the diagram $\mathcal{G}_{0} \leftarrow \mathcal{G}_{1} \rightarrow \mathcal{G}_{0}$, where the maps are the target and source maps and the actions are the left and right multiplication. A map $\mathcal{G}_{0} \leftarrow P \rightarrow \mathcal{H}_{0}$ is invertible (modulo the remark below) if both $s_{P}$ and $t_{P}$ are principal bundles.

Remark. The reader may note that composition is not associative on the nose but rather up to a natural notion of equivalence of maps (and similarly for identity maps and inverses). In Section 3 we will note some of the subtleties this causes, especially relating to gluing. For the most part these issues will not concern us. However as one reflection of this subtlety we prefer to call a map with $s_{P}, t_{P}$ both principal an equivalence rather than an isomorphism.

Given a strict homomorphism $\phi: \mathcal{G} \rightarrow \mathcal{H}$, there is an associated HS morphism given by

$$
\mathcal{G}_{0} \stackrel{\mathrm{pr}_{1}}{\longleftarrow} \mathcal{G}_{0} \times_{\phi, \mathcal{H}_{0}, t} \mathcal{H}_{1} \stackrel{s \circ \mathrm{pr}_{2}}{\longrightarrow} \mathcal{H}_{0}
$$

where the actions of $\mathcal{G}$ and $\mathcal{H}$ on $\mathcal{G}_{0} \times{ }_{\mathcal{H}_{0}} \mathcal{H}_{1}$ are given by $g \cdot(x, h) \cdot h^{\prime}=\left(t(g), \phi(g) h h^{\prime}\right)$. It is easy to check that this defines an HS morphism. The resulting HS morphism will be an equivalence exactly when the functor giving rise to it is an essential equivalence, defined as follows.

Definition-Proposition 2.7. A strict homomorphism $\phi: \mathcal{G} \rightarrow \mathcal{H}$ is an essential equivalence if one, hence both, of the following equivalent conditions are satisfied:

(1) the resulting HS map is an HS equivalence;

(2) (a) $\phi$ is essentially surjective: the map

$$
s \circ \mathrm{pr}_{2}: \mathcal{G}_{0} \times_{\phi, \mathcal{H}_{0}, t} \mathcal{H}_{1} \rightarrow \mathcal{H}_{0}
$$

is a surjective submersion;

(b) $\phi$ is fully faithful: the square

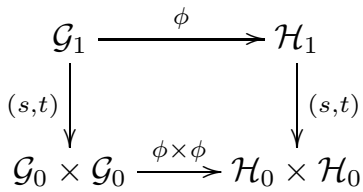

is a fiber product.

It is easy to check the equivalence of these conditions.

Note that the second condition implies that the underlying map of categories (without any topological or smooth structure) is an equivalence.

Remark. One can arrive at the notion of morphism of groupoids by inverting the essential equivalences (in the sense of localization of categories). However we prefer the HS definition, partly because it leads more easily to the "stacky" nature of groupoids; see Section [3. (See also [Pro96].) 
Example. We can let the source and the target of an HS morphism be translation groupoids. Let $X$ be a $K$-space and $Y$ an $L$-space, for Lie groups $K, L$.

Lemma 2.8. A morphism of translation groupoids $X \rtimes K \rightarrow Y \rtimes L$ is equivalent to the data of a $K$-equivariant principal $L$-bundle $s_{P}: P \rightarrow X$ over the $K$-space $X$ and a $K$-invariant and $L$-equivariant map $t_{P}: P \rightarrow Y$.

(Note: since $K$ acts on the right of $X$ and on the left of $P, s_{P}$ being $K$-equivariant means $s_{P}(k \cdot p)=s_{P}(p) \cdot k^{-1}$.

The simple proof is left to the reader. Note that if we understand $X$ and $Y$ to be given the trivial actions of $L$ and $K$ respectively, we can describe both $s_{P}$ and $t_{P}$ as $K^{\mathrm{op}} \times L$-equivariant maps.

We can further specialize by setting $Y$ to be a point, $Y=*$, and $K$ to be the trivial group, $K=(e)$. Then a map from $X=X \rtimes(e)$ to $\mathcal{B} L=* \rtimes L$ is simply a principal $L$-bundle over $X$. In other words, $\mathcal{B} L$ classifies $L$-bundles, as the notation suggests. (Note that this fits with the topological definition of the classifying space, which is $B G=E G / G$. $E G$ is contractible, hence "morally" a point; topologists use it instead of a point to get a "good" quotient. Here we actually use a point for the space of objects, but use an alternate category of "spaces" to take the quotient.) We will discuss this example further in Section 3 .

Many (but not all) properties and invariants of a groupoid are preserved by HS equivalence. For example, we have the following, saying that topological quotients and stabilizer groups are preserved.

Proposition 2.9. Let $\left(P, s_{P}, t_{P}\right)$ be an $H S$ map from $\mathcal{G}$ to $\mathcal{H}$. Then $P$ induces a continuous map on the underlying topological quotients, $f_{P}: \mathcal{G}_{\text {top }} \rightarrow \mathcal{H}_{\text {top }}$. Given $x \in \mathcal{G}_{0}$ and $y \in \mathcal{H}_{0}$ such that $f_{P}([x])=[y], P$ induces a group homomorphism $\psi_{P, x, y}: S_{x} \rightarrow S_{y}$, well defined up to conjugacy in the target.

If $P$ is an equivalence, then $f_{P}$ is a homeomorphism and each $\psi_{P, x, y}$ is an isomorphism.

Proof. The map $f_{P}: \mathcal{G}_{\text {top }} \rightarrow \mathcal{H}_{\text {top }}$ is defined by $f_{P}\left(\left[s_{P}(p)\right]\right)=\left[t_{P}(p)\right]$. Since $s_{P}$ is surjective, this defines $f_{P}$ for all $[x] \in \mathcal{G}_{\text {top }}$. To check that it is well defined, let $\left[s_{P}(p)\right]=\left[s_{P}(q)\right]$; then there is a $g: s_{P}(p) \rightarrow s_{P}(q)$ in $\mathcal{G}$, so $s_{P}(g \cdot p)=s_{P}(q)$. Since the $\mathcal{H}$-action on $P$ is principal, there is $h \in \mathcal{H}_{1}$ with $g \cdot p \cdot h=q$ and $h: t_{P}(q) \rightarrow t_{P}(g \cdot p)$. Since $t_{P}$ is $\mathcal{G}$-invariant, we have $h: t_{P}(q) \rightarrow t_{P}(p)$, so $\left[t_{P}(p)\right]=\left[t_{P}(q)\right]$. It is also straightforward to check that $f_{P}$ is continuous. If $P$ is an equivalence, then the construction works in both directions, and it is simple to check that the resulting maps are inverses.

Given $x \in \mathcal{G}_{0}, y \in \mathcal{H}_{0}$ with $f_{P}([x])=[y]$, we define $\psi_{P, x, y}: S_{x} \rightarrow S_{y}$ as follows. Let $p \in P$ with $s_{P}(p)=x$. We know there is $h_{0}: t_{P}(p) \rightarrow y$; fix such an $h_{0}$. Given $g \in S_{x}$, there is a unique $h \in \mathcal{H}_{1}$ with $p h=g p$; here $h: t_{P}(p)=t_{P}(g p)=$ $t_{P}(p h) \rightarrow t_{P}(p)$. Hence we define $\psi_{P, x, y}(g)=h_{0} h h_{0}^{-1} \in S_{y}$. It is simple to check that changing $p$ or $h_{0}$ changes the map $\psi_{P, x, y}$ by conjugating in the target. If $P$ is an equivalence, as above, the construction works in both directions to give inverse isomorphisms.

2.4. Orbifold groupoids. Moerdijk and Pronk (MP97, [Moe] show that to an effective orbifold with a given atlas, one can naturally associate a smooth proper effective étale groupoid. Equivalent atlases give rise to equivalent groupoids. Every such groupoid arises from an effective orbifold. Further, Ruan's good maps (Rua]) of orbifolds correspond to HS maps of groupoids. 
Hence instead of the traditional definition of orbifold, we will henceforth consider orbifolds as groupoids. More precisely, following Moerdijk, we will refer to a smooth proper étale groupoid as an orbifold groupoid, and refer to a smooth proper effective étale groupoid as an effective orbifold groupoid. (Actually Moerdijk allows the groupoid to be an arbitrary proper foliation groupoid, which is always equivalent to an étale groupoid.) Similarly, a map of orbifold groupoids (our replacement for the notion of a good map of orbifolds) will be a Hilsum-Skandalis map.

We remark that this gives an elegant answer to the question of exactly what conditions one should put on the ineffective parts of the stabilizers, which we alluded to in Section 1.1. One can translate the groupoid statement to one involving explicit charts, analogous to Definition [1.1, but it is not particularly enlightening. One of our main themes in this paper is to stay within the groupoid language and demonstrate its usefulness.

Henceforth we will freely use the word "orbifold" to mean "orbifold groupoid."

If we have presented orbifolds $M=X / K, N=Y / L$, then by Lemma 2.8 a map between the orbifolds is given by a $K$-equivariant principal $L$-bundle $s_{P}: P \rightarrow X$ and a $K$-invariant, $L$-equivariant map $t_{P}: P \rightarrow Y$.

\section{Groupoids as STACKS}

We want to point out some of the subtleties inherent in the definition of maps of orbifolds given in Section 2.3 and briefly relate orbifolds to stacks. This section can be skipped if the reader is only interested in the presentation problem. However it does serve as motivation for the next section on gerbes.

First, given two HS maps from $\mathcal{G}$ to $\mathcal{H}$, one can have an "isomorphism of HS maps" between them. It turns out that we do not want to simply identify two such isomorphic maps, as we would then lose the ability to glue maps. This leads to the idea of thinking of a groupoid as representing a stack. This point of view is very natural to algebraic geometers, but less so to differential geometers and topologists. However it should underlie all of the new "stringy" constructions on orbifolds. (See [LU] for more comments on this.)

Definition 3.1. Given two groupoids $\mathcal{G}$ and $\mathcal{H}$ and two HS maps $\left(P, s_{P}, t_{P}\right): \mathcal{G} \rightarrow$ $\mathcal{H}$ and $\left(Q, s_{Q}, t_{Q}\right): \mathcal{G} \rightarrow \mathcal{H}$, an isomorphism (or 2-isomorphism for definiteness) from $\left(P, s_{P}, t_{P}\right)$ to $\left(Q, s_{Q}, t_{Q}\right)$ is a $\mathcal{G} \times \mathcal{H}$-equivariant diffeomorphism $\alpha: P \rightarrow Q$ such that $s_{Q} \alpha=s_{P}$ and $t_{Q} \alpha=t_{P}$.

Hence for every $\mathcal{G}, \mathcal{H}$ there is a category (in fact, an abstract groupoid) $\operatorname{HS}(\mathcal{G}, \mathcal{H})$ of HS maps from $\mathcal{G}$ to $\mathcal{H}$.

One would like to say this makes (Groupoids, HS maps) into a 2-category, but that is not quite true. Since the definition of composition of HS maps involves taking a pullback, composition of morphisms is not strictly associative, but only associative up to a coherent isomorphism. Hence (Groupoids, HS maps) form what is called a bicategory. However the lack of strict associativity of composition will not bother us too much, so it is not too wrong to think of this as a 2-category.

It is tempting to identify any two isomorphic HS maps to get a set of equivalence classes of maps. This does form a category, and often this is good enough. However there is one fundamental operation that fails when one does this, namely gluing maps together. A simple example shows the basic problem.

As in the previous section, let $M$ be a manifold and let $K$ be a group and consider the category of HS maps from the trivial groupoid $M$ to the classifying 
groupoid $\mathcal{B} K$. We saw that $H S(M, \mathcal{B} K)$ is the category of $K$-principal bundles on $M$. Suppose that $M=\bigcup_{\alpha} U_{a}$ is an open cover of $M$. Then a $K$-bundle over $M$ is determined by the data of a bundle on each $U_{\alpha}$ and specific gluing isomorphisms on each $U_{\alpha} \cap U_{\beta}$, such that the cocycle condition is satisfied.

If we simply identified any two isomorphic HS maps, then we would lose these specific gluing isomorphisms, retaining only the knowledge that the bundles happen to be isomorphic. If the cover is fine enough, that is no information at all since all bundles are locally trivial. Also, there is no way to talk about compatibility of gluings.

Hence HS maps glue in a different way from ordinary maps of manifolds. We have the following setup.

Let $M$ be a manifold (considered as a trivial groupoid) and $\mathcal{G}$ be an arbitrary groupoid. Let $\mathcal{U}=\left\{U_{\alpha}\right\}$ be an open cover of $M$, and denote $U_{\alpha} \cap U_{\beta}$ by $U_{\alpha \beta}$.

Definition 3.2. A collection of HS maps

$$
\psi_{\alpha}: U_{\alpha} \rightarrow \mathcal{G}
$$

and 2-isomorphisms of HS maps

$$
\chi_{\alpha \beta}:\left.\left.\psi_{\alpha}\right|_{U_{\alpha \beta}} \rightarrow \psi_{\beta}\right|_{U_{\alpha \beta}}
$$

is called descent data if the $\chi_{\alpha \beta}$ satisfy the cocycle condition

$$
\chi_{\alpha \beta} \chi_{\beta \gamma} \chi_{\gamma \alpha}=1 \text {. }
$$

We will only consider descent data that are normalized, i.e. with

$$
\chi_{\beta \alpha}=\left(\chi_{\alpha \beta}\right)^{-1} \text { and } \chi_{\alpha \alpha}=1 .
$$

Descent data with respect to a given open cover form a groupoid, denoted $\operatorname{Desc}(\mathcal{U}, \mathcal{G})$. An isomorphism of descent data from $(\psi, \chi)$ to $\left(\psi^{\prime}, \chi^{\prime}\right)$ is given by

$$
\eta_{\alpha}: \psi_{\alpha} \rightarrow \psi_{\alpha}^{\prime}
$$

such that

$$
\left.\chi_{\alpha \beta}^{\prime} \circ \eta_{\alpha}\right|_{U_{\alpha \beta}}=\left.\eta_{\alpha}\right|_{U_{\alpha \beta}} \circ \chi_{\alpha \beta} .
$$

The proof of the following proposition exactly parallels the proof of the corresponding fact for principal bundles.

Proposition 3.3. Let $M$ be a manifold (considered as a trivial groupoid) and let $\mathcal{G}$ be a groupoid. Let $\mathcal{U}=\left\{U_{\alpha}\right\}$ be an open cover of $M$. For every $H S$ map $\psi: M \rightarrow \mathcal{G}$, restriction to the open cover induces an equivalence between the categories

$$
H S(M, \mathcal{G}) \cong \operatorname{Desc}(\mathcal{U}, \mathcal{G}) \text {. }
$$

In the algebraic geometry language, this says that a groupoid $\mathcal{G}$ naturally induces a stack on the category of manifolds. We present some further explanation of this idea and clarification of some confusing issues in a related paper Met.

\section{Gerbes}

We will be particularly interested in a very simple kind of orbifold groupoid $\mathcal{G}$, namely one whose corresponding effective orbifold $\mathcal{G}_{\text {eff }}$ is equivalent to a manifold.

Definition 4.1. A smooth étale groupoid $\mathcal{G}$ is purely ineffective (PI) if $S_{\mathcal{G}}^{0}=S_{\mathcal{G}}$, i.e. if every stabilizer arrow gives the germ of the identity on $\mathcal{G}_{0}$. 
Proposition 4.2. Let $\mathcal{G}$ be a purely ineffective orbifold groupoid. Then the quotient map $p: \mathcal{G}_{0} \rightarrow \mathcal{G}_{\text {top }}$ is étale and the quotient $\mathcal{G}_{\text {top }}$ is a smooth manifold. Moreover the induced map from $\mathcal{G}_{\mathrm{eff}}$ to $\mathcal{G}_{\text {top }}$ is an equivalence.

Proof. First note that the quotient map is open, since given an open set $U \subset \mathcal{G}_{0}$, $p^{-1} p(U)=s t^{-1}(U)$ is open since $s$ is an open map.

Further, note that $S=S^{0}$ is open in $\mathcal{G}_{1}$, by Proposition 2.4.

Let $x \in \mathcal{G}_{0}$. We will show that there is a neighborhood $W$ of $x$ such that for any arrow $g: y \rightarrow z, y, z \in W$, we must have $y=z$. Given such a $W,\left.p\right|_{W}$ is open and injective, hence a homeomorphism onto its (open) image. This will prove $p$ étale. It will follow that $\mathcal{G}_{\text {top }}$ is a smooth manifold (it is Hausdorff because it is a quotient by a proper equivalence relation).

Now we show the existence of $W$. Let $U$ be a neighborhood of $x$ whose closure is compact. Let $A=(s, t)^{-1}(\bar{U} \times \bar{U})$. Since $\mathcal{G}$ is proper, $A$ is compact. Let $B=A \backslash S$ which is also compact.

Suppose that there is a sequence of points $y_{i}, z_{i} \in U$ with arrows $h_{i}: y_{i} \rightarrow z_{i}$, such that both $\left\{y_{i}\right\}$ and $\left\{z_{i}\right\}$ converge to $x$, and $h_{i} \notin S$. We have $h_{i} \in B$ which is compact, so there is a convergent subsequence $h_{i_{j}} \rightarrow h \in B$ of $h_{i}$. This $h$ must be an arrow with $s(h)=t(h)=x$, so $h \in S$, a contradiction. Hence no such sequence can exist, so there must be some neighborhood $W$ of the form desired; hence $p$ is étale.

To show that the induced strict homomorphism, which we shall also call $p$, from $\mathcal{G}_{\text {eff }}$ to $\mathcal{G}_{\text {top }}$ is an equivalence, we need to verify conditions (2a), (2b) in DefinitionProposition 2.7 Essential surjectivity is clear since the map on objects is a surjective étale map, hence certainly a surjective submersion. The map $p$ is also fully faithful since for both groupoids, there is a unique arrow from any object to any other object.

As a corollary, we know when a groupoid is equivalent to a manifold. The answer is simple and standard.

Corollary 4.3. Given a smooth proper étale groupoid $\mathcal{G}$, there is a manifold $M$ whose associated groupoid is equivalent to $\mathcal{G}$ if and only if $S_{\mathcal{G}}$ consists only of identity arrows. In that case, $\mathcal{G}_{\text {top }}$ is a smooth manifold and is diffeomorphic to $M$.

Proof. Given a smooth étale groupoid with $S_{\mathcal{G}}$ consisting only of identity arrows, Proposition 4.2 implies that $\mathcal{G}_{\text {top }}$ is a smooth manifold. It is easy to see that $p$ satisfies condition (2) of the definition of an essential equivalence.

Conversely, since stabilizer groups are preserved by equivalence, if $\mathcal{G}$ is equivalent to a manifold, it must have no nonidentity stabilizers.

Proposition 4.2 says that a PI orbifold groupoid is not a singular space at all, but instead is a smooth manifold with some extra structure, given by the stabilizers of the groupoid. In fact the extra structure is a well-known one, at least to algebraic geometers. Such a groupoid corresponds to a gerbe on the underlying manifold $\mathcal{G}_{\text {top }}$.

We recall the definition of a gerbe $F$ on a space $M$. See [Gir71, Bry93, [LMB00] for more details. A gerbe is roughly a certain kind of "sheaf of categories." (More precisely, it is a certain kind of stack.)

Definition 4.4. Let $M$ be a topological space. A (strict) gerbe $F$ on $M$ is an assignment of an abstract groupoid $F(U)$ to every open set $U \subset M$ and a strict 
homomorphism of groupoids (a functor) $r_{V U}: F(U) \rightarrow F(V)$ for every inclusion $V \subset U$. Denote $r_{V U}(x)=\left.x\right|_{V}$ for $x \in \operatorname{Ob} F(U)$, and for $x, y \in \operatorname{Ob} F(U)$, denote the set of arrows in $F(U)$ from $x$ to $y$ by $F(x, y)$. This data is required to satisfy:

(1) $r_{W V} \circ r_{V U}=r_{W U}$ for $W \subset V \subset U$.

(2) Given $U \subset M$ and $x, y \in \mathrm{Ob} F(U)$, the assignment $V \mapsto F\left(\left.x\right|_{V},\left.y\right|_{V}\right)$ defines a sheaf (of sets).

(3) Suppose we are given an open cover $\mathcal{U}=\left\{U_{\alpha}\right\}$ of $U$ and descent data for $F$ over $\mathcal{U}$, i.e. $x_{\alpha} \in \mathrm{Ob} F\left(U_{\alpha}\right)$ and $\phi_{\alpha \beta}:\left.\left.x_{\alpha}\right|_{U_{\alpha} \cap U_{\beta}} \cong x_{\beta}\right|_{U_{\alpha} \cap U_{\beta}}$ such that $\phi_{\alpha \beta} \phi_{\beta \gamma}=\phi_{\alpha \gamma}$ (when restricted to $U_{\alpha} \cap U_{\beta} \cap U_{\gamma}$ ). Then there is an $x \in \mathrm{Ob} F(U)$ and $\psi_{\alpha}:\left.x\right|_{U_{\alpha}} \cong x_{\alpha}$ such that $\phi_{\alpha \beta} \psi_{\alpha}=\psi_{\beta}$ (over $U_{\alpha} \cap U_{\beta}$ ).

(4) There is an open cover $\mathcal{U}=\left\{U_{\alpha}\right\}$ of $M$ such that each category $F\left(U_{\alpha}\right)$ is nonempty.

(5) Given $U \subset M$ and $x, y \in \operatorname{Ob} F(U)$, there is an open cover $\mathcal{V}=\left\{V_{\alpha}\right\}$ of $U$ such that for every $\alpha,\left.\left.x\right|_{V_{\alpha}} \cong y\right|_{V_{\alpha}}$.

This definition amalgamates a number of more general concepts. Condition (1) says that $F$ is a presheaf of categories. Adding condition (21) says that $F$ is a "prestack." Adding condition (3) says that $F$ is in fact a stack. One refers to this condition by saying that all descent data are effective, i.e. they define objects of $F(U)$ as stated.

Condition (4) says that the categories $F(U)$ are locally nonempty, and condition (5) says that any two objects are locally isomorphic. These last two conditions say that the stack $F$ is in fact a gerbe.

Remark. Condition (1), that the restriction functors commute on the nose, makes this a strict gerbe. Usually one allows the restriction functors to commute up to a coherent natural isomorphism. However in our case the strict condition will be satisfied, which simplifies the notation. $\mathcal{G}_{\text {top. }}$.

Now suppose $\mathcal{G}$ is a purely ineffective orbifold groupoid with topological quotient

We define a corresponding gerbe $F$ over $\mathcal{G}_{\text {top }}$ as follows. Informally this is done as follows. Locally, over a small enough open set $U \subset \mathcal{G}_{\text {top }}$,

$$
\text { Ob } F(U)=\Gamma\left(U ; \mathcal{G}_{0}\right)=\left\{\text { sections of } p: \mathcal{G}_{0} \rightarrow \mathcal{G}_{\text {top }} \text { over } U\right\} .
$$

The arrows are defined locally, for two local sections $\sigma, \tau: U \rightarrow \mathcal{G}_{0}$, by

$$
\operatorname{Ar} F(U)(\sigma, \tau)=\Gamma\left(U ; \mathcal{G}_{1}\right)_{\sigma, \tau}=\left\{\gamma: U \rightarrow \mathcal{G}_{1} \mid s \circ \gamma=\sigma, t \circ \gamma=\tau\right\} .
$$

Informally, the arrows over $U$ are just the different ways of gluing the representatives of $U$ together.

Formally, we first define a sheaf of categories $\hat{F}$ by

$$
\begin{aligned}
\operatorname{Ob} \hat{F}(U) & =\left\{\text { sections of } p: \mathcal{G}_{0} \rightarrow \mathcal{G}_{\text {top }} \text { over } U\right\}, \\
\operatorname{Ar} \hat{F}(U)(s, t) & =\left\{\gamma: U \rightarrow \mathcal{G}_{1} \mid s \circ \gamma=\sigma, t \circ \gamma=\tau\right\}
\end{aligned}
$$

and then take $F$ to be the associated stack. This is defined as follows (following [LMB00]). An object of $F(U)$ consists of a cover $\mathcal{U}=\left\{U_{\alpha}\right\}$ of $U$, and descent data over $\mathcal{U}$, i.e. a family $x_{\alpha} \in \hat{F}\left(U_{\alpha}\right)=\Gamma\left(U_{\alpha} ; \mathcal{G}_{0}\right)$, and morphisms

$$
f_{\alpha \beta}:\left.\left.x_{\beta}\right|_{U_{\alpha} \cap U_{\beta}} \rightarrow x_{\alpha}\right|_{U_{\alpha} \cap U_{\beta}}
$$

satisfying $f_{\alpha \beta} f_{\beta \gamma}=f_{\alpha \gamma}$. By the definition of $\hat{F}, f_{\alpha \beta}$ is a section of $\mathcal{G}_{1}$ over $U_{\alpha} \cap U_{\beta}$. 
An arrow of $F(U)$ from $\left(\left\{U_{\alpha}\right\},\left\{x_{\alpha}\right\},\left\{f_{\alpha \beta}\right\}\right)$ to $\left(\left\{U_{\gamma}^{\prime}\right\},\left\{x_{\gamma}^{\prime}\right\},\left\{f_{\gamma \delta}^{\prime}\right\}\right)$ is given by the following data. Let $V_{\alpha \gamma}=U_{\alpha} \cap U_{\gamma}^{\prime}$; these form a cover of $U$ which refines both $\left\{U_{\alpha}\right\}$ and $\left\{U_{\gamma}^{\prime}\right\}$. Then an arrow consists of a family of morphisms $\left\{g_{\gamma \alpha}\right\}$ of $\hat{F}\left(V_{\alpha \gamma}\right)$ from $\left.x_{\alpha}\right|_{V_{\alpha \gamma}}$ to $\left.x_{\gamma}^{\prime}\right|_{V_{\alpha \gamma}}$, compatible with the descent isomorphisms:

$$
g_{\gamma \alpha} f_{\alpha \beta}=f_{\gamma \delta}^{\prime} g_{\delta \beta} .
$$

(This is defined over $U_{\alpha} \cap U_{\beta} \cap U_{\gamma}^{\prime} \cap U_{\delta}^{\prime}$.)

Restriction of objects and arrows from $F(U)$ to $F(V)$, for $V \subset U$, is defined by restriction of $x_{\alpha}$, etc., in the obvious way.

It is straightforward to verify that this satisfies conditions (11)-(3) of Definition 4.4

By Proposition 4.2, $\mathcal{G}_{0} \rightarrow \mathcal{G}_{\text {eff }}=\mathcal{G}_{\text {top }}$ always has local sections (being étale), verifying condition (4). Further, the map $\mathcal{G}_{1} \rightarrow \mathcal{G}_{0}$ has local sections, and hence any two objects of $F(U)$ are locally isomorphic, verifying condition (5). Therefore $F$ is indeed a gerbe. Note that if there are effective stabilizers, one will not get local sections of $\mathcal{G}_{0} \rightarrow \mathcal{G}_{\text {top }}$, so one does not obtain a gerbe over $\mathcal{G}_{\text {top }}$.

Conversely, if we start with a smooth manifold $M$ and a certain type of gerbe $F$ on $M$ (one whose band is locally constant, cf. Definition 5.4 below, and which has finite isotropy groups) one can construct a corresponding orbifold groupoid $\mathcal{G}=(M, F)$. In fact there is an equivalence between orbifolds with only ineffective stabilizers and these types of gerbes over manifolds. We will not need this result here.

\section{Presentations of orbifolds}

In this section we will prove a number of propositions that reduce the presentation problem for a general orbifold groupoid to a particular problem about equivariant abelian gerbes. We also show that the corresponding nonequivariant problem is solvable.

First we desingularize the space using the standard procedure of passing to the frame bundle.

Let $\mathcal{G}$ be a smooth étale groupoid of dimension $n$. Let $F=\operatorname{Fr}\left(T \mathcal{G}_{0}\right)$ be the orthonormal frame bundle of $\mathcal{G}_{0}$. Then there is a natural left action of $\mathcal{G}$ on $F$ with the standard projection $\pi: F \rightarrow \mathcal{G}_{0}$ as the base map. For, since $\mathcal{G}$ is étale, given an arrow $g: x \rightarrow y$ and a frame $f=\left(e_{1}, \ldots, e_{n}\right)$ with each $e_{k} \in T_{y}\left(\mathcal{G}_{0}\right)$, we can use the induced germ of a diffeomorphism $\phi_{g}$ to act on the frame: $g \cdot f=\phi_{g}(f)$. This is easily seen to be a smooth left action of $\mathcal{G}$. Further, this action commutes with the natural right action of $O(n)$ on $F$.

We can therefore use the following, which is stated with an extra group action present for later use.

Lemma 5.1. Let $\mathcal{G}$ be a smooth groupoid and let $K, L$ be Lie groups. Let $\pi: F \rightarrow$ $\mathcal{G}_{0}$ be a left $\mathcal{G}$-equivariant, right $L$-principal bundle over $\mathcal{G}_{0}$, and assume that the actions of $\mathcal{G}$ and $L$ commute. Further, assume that $K$ acts on $\mathcal{G}$ and on $F, \pi$ is $K$ equivariant, the actions of $K$ and $L$ commute, and the actions of $\mathcal{G}$ and $K$ satisfy

$$
(g \cdot f) \cdot k=(g \cdot k) \cdot(f \cdot k)
$$

for $g \in \mathcal{G}_{1}, f \in F, k \in K$. Then $K \times L$ acts on the right of $\mathcal{G} \ltimes F$ and the map $\pi$ induces a $K$-equivariant equivalence between $(\mathcal{G} \ltimes F) \rtimes L$ and $\mathcal{G}$. 
Proof. It is clear that $K \times L$ acts on the right of $\mathcal{G} \ltimes F$, by $(g, f) \cdot(k, l)=(g \cdot k,(f \cdot k) \cdot l)$. We have

$$
((\mathcal{G} \ltimes F) \rtimes L)_{0}=F, \quad((\mathcal{G} \ltimes F) \rtimes L)_{1}=\mathcal{G}_{1} \times_{s, \mathcal{G}_{0}, \pi} F \times L .
$$

We extend $\pi: F \rightarrow \mathcal{G}_{0}$ to arrows by defining $\pi(g, f, l)=g$. As usual this defines a smooth covariant functor, i.e. a strict homomorphism of groupoids. On objects, $\pi$ is a surjective submersion, since it is a principal bundle. We also need to check that $\pi$ is fully faithful. Since $F$ is an $L$-principal bundle, we have $F \times L \cong F \times{ }_{\mathcal{G}_{0}} F$. Hence

$$
\begin{aligned}
\mathcal{G}_{1} \times \mathcal{G}_{0} F \times L & \cong \mathcal{G}_{1} \times \times_{\mathcal{G}_{0}} F \times \mathcal{G}_{0} F \\
& \cong \mathcal{G}_{1} \times{ }_{\mathcal{G}_{0} \times \mathcal{G}_{0}}(F \times F)
\end{aligned}
$$

which says that $\pi$ is fully faithful.

Hence $\pi$ is an essential equivalence. It is also clearly $K$-equivariant.

Note that $\mathcal{G} \ltimes F$ should be thought of as a principal $L$-bundle over $\mathcal{G}$. Also, we want to think of $(\mathcal{G} \ltimes F) \rtimes L$ as (a finer model of) the quotient of $\mathcal{G} \ltimes F$ by the action of $L$. So the lemma can be thought of as simply saying that when we mod out the total space of an $L$-bundle by $L$, we recover the base space.

Our first application of the lemma is the following.

Proposition 5.2. Let $\mathcal{G}$ be an orbifold groupoid of dimension n. Put a $\mathcal{G}$-invariant Riemannian metric on $\mathcal{G}_{0}$ and let $\pi: F=\operatorname{Fr}\left(T \mathcal{G}_{0}\right) \rightarrow \mathcal{G}_{0}$ be the orthonormal frame bundle of $\mathcal{G}_{0}$. Then $\mathcal{G} \ltimes F$ is a purely ineffective groupoid with a right action of $O(n)$, and $\pi$ induces an $O(n)$-equivariant equivalence $(\mathcal{G} \ltimes F) \rtimes O(n) \cong \mathcal{G}$. Further, $S_{(\mathcal{G} \ltimes F)}^{0} \cong \pi^{*}\left(S_{\mathcal{G}}^{0}\right)$.

Proof. The action of $O(n)$ and the stated equivalence follow directly from the lemma (with $K$ trivial). We need only show that $\mathcal{G} \ltimes F$ is purely ineffective. Given $x \in \mathcal{G}_{0}$, $f=\left(e_{1}, \ldots, e_{n}\right), e_{k} \in T_{x} \mathcal{G}_{0}$, and $g \in \mathcal{G}_{1}, g: x \rightarrow y$, suppose that $(g, f) \in S_{(\mathcal{G} \ltimes F)}$, i.e. that $g \cdot f=f$. Then $\left(\phi_{g}\right)_{*}=1$ on $T_{x} \mathcal{G}_{0}$. Now $g \in S_{\mathcal{G}}(x)$ which is a finite group, so $\phi_{g}$ must be the identity. (Use an invariant inner product on a neighborhood of $x$ in $\mathcal{G}_{0}$ and the resulting exponential map.) Hence $g \in S_{\mathcal{G}}^{0}$, and $(g, f) \in S_{(\mathcal{G} \ltimes F)}^{0}$. Hence $S_{(\mathcal{G} \ltimes F)}=S_{(\mathcal{G} \ltimes F)}^{0} \cong \pi^{*}\left(S_{\mathcal{G}}^{0}\right)$.

In particular, if $\mathcal{G}$ is effective, then $\mathcal{G} \ltimes F$ has no stabilizers, so is equivalent to a manifold. This is the groupoid version of the proof that an effective orbifold is presentable as the quotient of its frame bundle by $O(n)$.

Now we reduce the complexity of $\mathcal{G}$ at the expense of making the compact group larger. Given a purely ineffective orbifold groupoid $\mathcal{G}$, the stabilizers will not generally be abelian, and the local system $S_{\mathcal{G}}^{0}$ may not be $\mathcal{G}$-equivariantly trivial. We will replace $\mathcal{G}$ with a new groupoid $\mathcal{G}^{\prime}$, where $S_{\mathcal{G}^{\prime}}^{0}$ is abelian and $\mathcal{G}$-equivariantly trivial, using a standard trick from bundle theory. We will assume that $\mathcal{G}$ is connected (i.e. $\mathcal{G}_{\text {top }}$ is connected) for simplicity.

Proposition 5.3. Let $\mathcal{G}$ be a purely ineffective connected orbifold groupoid and let $K$ be a Lie group acting on $\mathcal{G}$ on the right. Let $T=S_{\mathcal{G}}^{0}(x)$ for some $x \in \mathcal{G}_{0}$. Then there is a purely ineffective orbifold groupoid $\mathcal{G}^{\prime}$ with an action of $K \times \operatorname{Aut}(T)$ such that $\mathcal{G}^{\prime} \rtimes \operatorname{Aut}(T)$ is $K$-equivariantly equivalent to $\mathcal{G}$ and the stabilizer local system $S_{\mathcal{G}^{\prime}}^{0}$ of $\mathcal{G}^{\prime}$ is $\left(\mathcal{G}^{\prime}, K \times \operatorname{Aut}(T)\right)$-equivariantly isomorphic to $\mathcal{G}_{0}^{\prime} \times Z(T)$. In particular the stabilizer local system of $\mathcal{G}^{\prime}$ is abelian and $K$-equivariantly trivial. 
Proof. Let

$$
F=\left\{(x, \phi) \in \mathcal{G}_{0} \times \operatorname{Hom}(T, \mathcal{G}) \mid \phi: T \cong S_{\mathcal{G}}^{0}(x)\right\} .
$$

There is an obvious projection map $\pi: F \rightarrow \mathcal{G}_{0}$. It is surjective since all of the stabilizer groups of $\mathcal{G}$ are isomorphic to $T$ (since $\mathcal{G}_{\text {top }}$ is connected and $S_{\mathcal{G}}^{0}$ is a local system). In fact it makes $F$ into a $\mathcal{G}$-equivariant $\operatorname{Aut}(T)$-principal bundle, where the left action of $(g: x \rightarrow y) \in \mathcal{G}$ is

$$
g \cdot(x, \phi)=\left(y, g \phi g^{-1}\right)
$$

and the right action of $\lambda \in \operatorname{Aut}(T)$ is

$$
(x, \phi) \cdot \lambda=(x, \phi \circ \lambda) \text {. }
$$

These actions clearly commute. It is also easy to check that everything is $K$ equivariant. Hence by Lemma [5.1, $K \times \operatorname{Aut}(T)$ acts on the right of $\mathcal{G} \ltimes F$ and $\pi$ induces a $K$-equivariant equivalence between $(\mathcal{G} \ltimes F) \rtimes \operatorname{Aut}(T)$ and $\mathcal{G}$. So we let $\mathcal{G}^{\prime}=\mathcal{G} \ltimes F$.

It is clear that $S_{\mathcal{G}^{\prime}}^{0}=S_{\mathcal{G}^{\prime}}$, i.e. that the $\mathcal{G}^{\prime}$ is purely ineffective, since $\mathcal{G}$ is.

By definition, $\mathcal{G}_{0}^{\prime}=F$, and an arrow of $\mathcal{G}^{\prime}$ from $(x, \phi)$ to $(y, \psi)$ is an arrow $g: x \rightarrow y$ in $\mathcal{G}_{1}$ such that $\psi=g \phi g^{-1}$, i.e. $\psi(t)=g \phi(t) g^{-1}$ for every $t \in T$. Hence the stabilizers are

$$
S_{\mathcal{G}^{\prime}}^{0}(x, \phi)=\left\{g: x \rightarrow x \mid \phi(t)=g \phi(t) g^{-1} \forall t \in T\right\}=Z\left(S_{\mathcal{G}}^{0}(x)\right)
$$

since each $\phi$ is surjective. Define a map $c: \mathcal{G}_{0}^{\prime} \times Z(T) \rightarrow S_{\mathcal{G}^{\prime}}^{0}$ by $c((x, \phi), a)=\phi(a)$. More precisely, we should remember the source and target of this arrow, which are both equal to $(x, \phi)$; hence where necessary we will denote the right-hand side by

$$
c((x, \phi), a)=\phi(a)_{(x, \phi)} .
$$

By (5.2), this defines an isomorphism of local systems over $\mathcal{G}_{0}^{\prime}$. It is $\mathcal{G}^{\prime}$-equivariant since, for $g:(x, \phi) \rightarrow(y, \psi)$,

$$
g c((x, \phi), a) g^{-1}=g \phi(a) g^{-1}=\psi(a)=c((y, \psi), a) .
$$

It is also $\operatorname{Aut}(T)$-equivariant, where $\operatorname{Aut}(T)$ acts on the right of $Z(T)$ by $a \cdot \lambda=$ $\lambda^{-1}(a)$. Recall that $\operatorname{Aut}(T)$ acts on $\mathcal{G}_{0}^{\prime}$ as in (5.1); we have

$$
c((x, \phi), a) \cdot \lambda=\phi(a)_{(x, \phi)} \cdot \lambda=\phi(a)_{(x, \phi \circ \lambda)}
$$

and

$$
c((x, \phi) \cdot \lambda, a \cdot \lambda)=c\left((x, \phi \circ \lambda), \lambda^{-1}(a)\right)=\phi(a)_{(x, \phi \circ \lambda)} .
$$

It is also easy to check that $c$ is $K$-equivariant. Hence we have a $K$-equivariant trivialization of the stabilizer local system of $\mathcal{G}^{\prime}$, and this local system is abelian.

Note: in gerbe language, this proposition accomplishes two things. It reduces to the case of an abelian gerbe, and it reduces to the case of a trivial band:

Definition 5.4. A gerbe $F$ on a manifold $M$, represented by a purely ineffective groupoid $\mathcal{G}$, has trivial abelian band $A$, where $A$ is an abelian group, if the stabilizer local system $S_{\mathcal{G}}^{0}$ of $\mathcal{G}$ is $\mathcal{G}$-equivariantly isomorphic to $\mathcal{G}_{0} \times A$ for some abelian group $A$.

In fact one can quite generally define the notion of the band of a gerbe, which is analogous to the coefficient sheaf for a torsor, but it is somewhat involved. See Giraud Gir71 for the original definition. 
Combining the two previous propositions gives the following almost-presentation theorem.

Theorem 5.5. Let $\mathcal{G}$ be a connected orbifold groupoid of dimension $n$. Then $G$ can be presented as $\mathcal{G} \cong \mathcal{H} / K$, where $\mathcal{H}$ is a purely ineffective orbifold groupoid (i.e. a manifold $P$ together with a gerbe $F$ on $P$ ) with trivial finite abelian band and an action of the compact Lie group $K$. The stabilizer groups of $\mathcal{H}$ are isomorphic to $Z(T)$, where $T$ is the ineffective stabilizer of some point of $\mathcal{G}_{0}$.

As a corollary we obtain a result of Edidin-Hassett-Kresch-Vistoli [EHKV]:

Corollary 5.6. Let $\mathcal{G}$ be a connected orbifold groupoid such that the ineffective stabilizers all have trivial center. Then $\mathcal{G}$ can be presented as $\mathcal{G} \cong P / K$, where $P$ is a smooth $K$-manifold and $K$ is a compact Lie group.

We are thus led to consider the following situation. Let $A$ be a finite abelian group and let $\mathcal{G}$ be a purely ineffective orbifold groupoid such that the stabilizer local system $S_{\mathcal{G}}^{0}$ of $\mathcal{G}$ is $\mathcal{G}$-equivariantly isomorphic to $\mathcal{G}_{0} \times A$. I.e., we have a smooth manifold $M=\mathcal{G}_{\text {top }}$ and a gerbe $F$, with trivial abelian band.

Theorem 5.7. Given a smooth m-manifold $M$ and a gerbe $F$, with trivial finite abelian band $A$. Then $(M, F)$ can be presented as the quotient $P / K$ of a smooth $K$-manifold $P$, where $K$ is a compact Lie group; in fact $K$ can be taken to be a finite product of unitary groups, with $A$ centrally embedded in $K$, and $P$ can be taken to be the total space of a principal $K / A$ bundle.

We prove a preliminary result before proving this theorem. Let $A \cong \mathbb{Z} / a \mathbb{Z}$ be a cyclic group and let $i: A \rightarrow U(n)$ be a central embedding of $A$ into a unitary group. The exact sequence

$$
0 \longrightarrow A \stackrel{i}{\longrightarrow} U(n) \longrightarrow U(n) / A \longrightarrow 0
$$

gives rise to a fibration sequence

$$
B U(n) \longrightarrow B(U(n) / A) \stackrel{\beta}{\longrightarrow} K(A, 2),
$$

where $\beta$ is the Bockstein map for the sequence.

For integers $n, k$, let $d_{n, k}: U(n) \rightarrow U(n)^{k} \rightarrow U(n k)$ be the composition of the diagonal map and the natural embedding of $U(n)^{k}$ as block diagonal matrices in $U(n)$. These maps form a direct system of groups and we define $U(\infty)$ to be the direct limit of this system. (Note that this is different from $U$, which is the direct limit under direct sum with an identity matrix.) Note that the sequence $U(1) \rightarrow \ldots \rightarrow U(n !) \rightarrow U((n+1) !) \rightarrow \ldots$ is cofinal in our direct system, so $U(\infty)=\lim U(n !)$.

The maps $d_{n, k}$ preserve the center, so given a central embedding $i: A \rightarrow U(n)$ for some $n$, there will be a corresponding central embedding in any $U(n k)$, and hence an embedding $i_{\infty}: A \rightarrow U(\infty)$. We have an exact sequence

$$
0 \rightarrow A \rightarrow U(\infty) \rightarrow U(\infty) / A \rightarrow 0
$$

The following result makes more precise a result of Serre, cited in Gro68.

Proposition 5.8. Let $A$ be a cyclic group and let $S \subset K(A, 2)$ be an $m$-dimensional skeleton. Then there is an integer $n=n_{m}$ and a central embedding $i: A \rightarrow U(n)$ 
such that the associated Bockstein map $\beta: B(U(n) / A) \rightarrow K(A, 2)$ has a section over $S$.

Proof. We construct the section using obstruction theory. First let $n_{0}=1$, and let $i: A \rightarrow U(1)$ be the standard embedding. We will try to section the map $\beta_{0}: B\left(U\left(n_{0}\right) / A\right) \rightarrow K(A, 2)$ over successive skeleta of $S$, allowing ourselves to make $n_{0}$ larger, using the maps $d_{n, k}$ above, as follows. We will define a sequence

$$
U\left(n_{0}\right) \underset{d_{n_{0}, q_{0}}}{\longrightarrow} U\left(n_{1}\right) \underset{d_{n_{1}, q_{1}}}{\longrightarrow} U\left(n_{2}\right) \underset{d_{n_{2}, q_{2}}}{\longrightarrow} \cdots
$$

(where $n_{k}=q_{k-1} n_{k-1}$ ). Each term $U\left(n_{k}\right)$ in the sequence has its associated map $\beta_{k}: B\left(U\left(n_{k}\right) / A\right) \rightarrow K(A, 2)$, and these maps commute with the $d$ maps as noted above. We will inductively find a section of each $\beta_{k}$ over the $k$-skeleton of $S$.

Clearly there is a section $s_{0}$ of $\beta_{0}: B\left(U\left(n_{0}\right) / A\right) \rightarrow K(A, 2)$ over the 0 -skeleton of $K(A, 2)$. Now suppose that we have defined $q_{j}$ for all $j<k$ (which defines $n_{k}$ ) and we have found a section $s_{k}$ of $\beta_{k}: B\left(U\left(n_{k}\right) / A\right) \rightarrow K(A, 2)$ over the $k$-skeleton of $K(A, 2)$. The obstruction to extending the section of $\beta_{k}$ over the $k+1$-skeleton lies in

$$
H^{k+1}\left(K(A, 2) ; \pi_{k}\left(B U\left(n_{k}\right)\right)\right) \cong H^{k+1}\left(K(A, 2) ; \pi_{k-1}\left(U\left(n_{k}\right)\right)\right) .
$$

It is well known that any element of $H^{k+1}(K(A, 2) ; B)$ is torsion, for a finite abelian group $A$ and an arbitrary abelian group $B$. So let $q_{k}$ be the least integer such that every element of $H^{k+1}\left(K(A, 2) ; \pi_{k-1}\left(U\left(n_{k}\right)\right)\right)$ is $q_{k}$-torsion. So in particular the obstruction class is $q_{k}$-torsion. It is easy to see that under the diagonal map $d=d_{n_{k}, q_{k}}$, this class dies. Hence we can extend the section $d_{n_{k}, q_{k}} s_{k}$ of $\beta_{k+1}$ over the $k+1$-skeleton of $K(A, 2)$. Continuing in this way, we get a section of $\beta_{m}: B\left(U\left(n_{m}\right) / A\right) \rightarrow K(A, 2)$ over the $m$-skeleton, where $n_{m}=n_{0} \cdot \prod_{k=0}^{m-1} q_{k}$.

(Note: since $B U\left(n_{0}\right)$ is 1-connected, we have $q_{0}=q_{1}=1$ and so $n_{2}=n_{0}$. Higher $q_{k}$ values are more complicated but calculable in principle.)

Now the proof of the theorem is easy.

Proof of Theorem 5.7. First we prove the theorem in the case where $A$ is cyclic. The gerbe $F$ is classified by an element $\alpha \in H^{2}(M ; A)$. The class $\alpha$ defines a map $\alpha: M \rightarrow K(A, 2)$. Since $M$ is an $m$-manifold we can assume that $\alpha$ maps into an $m$-skeleton $S$ of $K(A, 2)$. Then Proposition 5.8 guarantees a lift of $\alpha$ through the Bockstein map associated to some embedding $A \rightarrow U(n)$. This lift classifies a $U(n) / A$-bundle $P$ whose Bockstein is $\alpha$. But for any such bundle we know that the class of the associated $A$-gerbe is given by the Bockstein of the classifying map of $P$. Hence we can present the $A$-gerbe using $P$.

If $A$ is an arbitrary finite abelian group, express it as a product $A=\prod A_{k}$ and apply the above argument to each factor. We get a presentation with $K=\prod U\left(n_{k}\right)$ and $P=P_{1} \times_{M} P_{2} \cdots \times_{M} P_{r}$.

Note that for $A$ cyclic, we can take $n=n_{m}$ as in the proof of the last proposition. This gives, in principle, a computable bound for how large the group $U(n)$ must be.

In order to use this method to solve the general presentation problem for orbifolds, one needs a positive answer to following equivariant version of Theorem 5.7

Question. Let $G$ be a purely ineffective orbifold groupoid with equivariantly trivial abelian stabilizers, i.e. let $G$ represent an abelian gerbe $F$ with finite stabilizers 
and trivial band $A$ on a smooth manifold $M$. Let $K$ be a compact Lie group and assume that $K$ acts (strictly) on the right of $G$. Can we find a smooth manifold $P$, a compact Lie group $L$, and an action of $K \times L$ on $P$ such that we have a $K$-equivariant equivalence

$$
P \rtimes L \cong G ?
$$

\section{REFERENCES}

[AR] Alejandro Adem and Yongbin Ruan. Twisted Orbifold K-Theory. Contemporary Mathematics, 310. American Mathematical Society, Providence, RI, 2002. MR 2003g:00020

[Bry93] Jean-Luc Brylinski. Loop spaces, characteristic classes and geometric quantization, volume 107 of Progress in Mathematics. Birkhäuser Boston Inc., Boston, MA, 1993. MR 94b:57030

[CR] Weimin Chen and Yongbin Ruan. A New Cohomology Theory for Orbifold. Preprint, available as arXiv:math.AG/0004129.

[Cra99] Marius Crainic. Cyclic cohomology of étale groupoids: the general case. K-Theory, 17(4):319-362, 1999. MR 2000j:19002

[EHKV] D. Edidin, B. Hassett, A. Kresch, and A. Vistoli. Brauer groups and quotient stacks. Amer. J. Math. 123(4):761-777, 2001. MR 2002f:14002

[Gir71] Jean Giraud. Cohomologie non abélienne. Springer-Verlag, Berlin, 1971. Die Grundlehren der mathematischen Wissenschaften, Band 179. MR 49:8992

[Gro68] Alexander Grothendieck. Le groupe de Brauer. I. Algèbres d'Azumaya et interprétations diverses. In Dix Exposés sur la Cohomologie des Schémas, pages 46-66. North-Holland, Amsterdam, 1968. MR 39:5586a

[Hen] Andre Henriques. Orbispaces and Orbifolds from the Point of View of the Borel Construction, a new Definition.

[Kaw78] Tetsuro Kawasaki. The signature theorem for V-manifolds. Topology, 17:75-83, 1978. MR 57:14072

[Kaw79] Tetsuro Kawasaki. The Riemann-Roch theorem for complex V-manifolds. Osaka J. Math., 16:151-159, 1979. MR 80f:58042

[LMB00] Gérard Laumon and Laurent Moret-Bailly. Champs algébriques, volume 39 of Ergebnisse der Mathematik und ihrer Grenzgebiete. 3. Folge. A Series of Modern Surveys in Mathematics [Results in Mathematics and Related Areas. 3rd Series. A Series of Modern Surveys in Mathematics]. Springer-Verlag, Berlin, 2000. MR 2001f:14006

[LU] Ernesto Lupercio and Bernardo Uribe. Gerbes over Orbifolds and Twisted K-theory. Preprint, available as arXiv:math.AT/0105039.

[Met] David Metzler. Topological stacks, gerbes, groupoids, and orbispaces. in preparation.

[Moe] Ieke Moerdijk. Orbifolds as Groupoids: an Introduction. Orbifolds in mathematics and physics (Madison, WI, 2001), 205-222, Contemp. Math. 310, Amer. Math. Soc., Providence, RI, 2002.

[MP97] I. Moerdijk and D. A. Pronk. Orbifolds, sheaves and groupoids. K-Theory, 12(1):3-21, 1997. MR 98i:22004

[Pro96] Dorette A. Pronk. Etendues and stacks as bicategories of fractions. Compositio Math., 102(3):243-303, 1996. MR 97d:18011

[Rua] Yongbin Ruan. Stringy Geometry and Topology of Orbifolds. Preprint, available as arXiv:math.AG/0011149.

[Sat56] Ichiro Satake. On a generalization of the notion of manifold. Proc. Nat. Acad. Sci. USA, 42:359-363, 1956. MR 18:144a

Department of Mathematics, Massachusetts Institute of Technology, Cambridge, MASSACHUSETTS 02139

E-mail address: andrhenr@mit.edu

Department of Mathematics, University of Florida, Gainesville, Florida 32611

E-mail address: metzler@math.ufl.edu 